\title{
Automated Tetrahedral Mesh Generation for CFD Analysis of Aircraft in Conceptual Design
}

\author{
Irian Ordaz ${ }^{1}, \mathrm{Wu} \mathrm{Li}^{2}$, and Richard Campbell ${ }^{3}$ \\ NASA Langley Research Center, Hampton, Virginia 23681, USA
}

\begin{abstract}
The paper introduces an automation process of generating a tetrahedral mesh for computational fluid dynamics (CFD) analysis of aircraft configurations in early conceptual design. The method was developed for CFD-based sonic boom analysis of supersonic configurations, but can be applied to aerodynamic analysis of aircraft configurations in any flight regime.
\end{abstract}

\section{Nomenclature}

$\begin{array}{ll}\alpha & =\text { angle of attack } \\ d p / p & =\text { non-dimensional pressure } \\ \phi & =\text { azimuthal angle } \\ H & =\text { total height (or diameter) of the half-cylindrical computational domain } \\ H_{\text {far }} & =\text { height of the configuration from the under-track } \\ N_{\mathrm{a}} & =\text { number of points along the azimuthal direction } \\ N_{\text {lower }} & =\text { number of points along the under-track } \\ N_{\mathrm{r}} & =\text { number of points along the radial direction } \\ N_{\text {upper }} & =\text { number of points along the over-track } \\ R & =\text { radius of the half-cylindrical computational domain } \\ X_{\text {aft }} & =\text { aft distance of the half-cylinder from the end of the configuration } \\ X_{\text {fore }} & =\text { fore distance of the half-cylinder from the nose of the configuration } \\ \text { CAD } & =\text { computer-aided design } \\ \text { CFD } & =\text { computational fluid dynamics } \\ \text { VSP } & =\text { Vehicle Sketch Pad }\end{array}$

\section{Introduction}

$\mathrm{T}$ HIS paper documents an automation process from a conceptual geometry model in Vehicle Sketch Pad $^{1}$ (VSP) to computational fluid dynamics (CFD) analysis of the geometry using tetrahedral meshes. The process uses VSP's surface meshing capability, the 2-D and 3-D meshing capabilities of AFLR2 and AFLR3 ${ }^{2}$ (AdvancingFront/Local-Reconnection), and a knowledge-based tetrahedral mesh extrusion method ${ }^{3}$ for efficient off-body pressure calculation. Using this process one can evaluate the sonic boom characteristics of any VSP geometry model by using unstructured CFD solvers (such as FUN3D ${ }^{4}$ and USM3D ${ }^{5}$ ) in a few hours with minimal manual setup. With the existing in-house automation process ${ }^{6,7}$ from VSP geometry to Cartesian CFD analysis using Cart3D ${ }^{8}$; it is easy to compare the solutions generated by various CFD codes for the same VSP geometry model. These automated CFD analysis processes allow a quick study of code dependency for sonic boom analysis of a supersonic aircraft.

Tetrahedral meshing tools (such as Gridgen ${ }^{9}$ and VGRID $^{10}$ ) usually require a CAD-like solid model definition of the geometry as the input. In the conceptual design phase, when the intersection curves between two components are implicitly defined by the intersection of the two surfaces, it is labor intensive to construct the required input data (such as connectors) for tetrahedral meshing. An earlier effort to bridge the gap between a conceptual geometry and tetrahedral meshing was documented in Ref. 11, with two limitations on the complexity of the geometry models. These two limitations are: (1) three surfaces cannot intersect at one point and (2) the intersection curve between two components forms a closed loop on each component. Moreover, giving a set of required inputs for a tetrahedral

\footnotetext{
${ }^{1}$ Aerospace Engineer, Aeronautics Systems Analysis Branch

${ }^{2}$ Senior Research Engineer, Aeronautics Systems Analysis Branch

${ }^{3}$ Senior Research Engineer, Configuration Aerodynamics Branch, AIAA Associate Fellow
} 
meshing tool, there is no guarantee that a volume mesh will be successfully created. For in-depth CFD applications, flexible control on cell size at different locations on the geometry surface is very important to capture the flow physics without using an intractable amount of cells. However, meshing tools that allow flexible control on cell size are less user-friendly and might be more prone to failure due to "improper" sourcing inputs [that control the cell size] specified by novice users. AFLR3 is a robust tetrahedral meshing tool for CFD analysis which only requires a user to provide a boundary mesh of reasonable quality for the computational domain.

\section{Automation Process of Inner Mesh Boundary}

The automation process begins with a high quality triangulated surface mesh generated with VSP's CFD meshing capability as described in Ref. 12. Alternatively, a triangulated surface mesh from a third-party tool can be used as long as it is of reasonable quality for volume mesh generation with AFLR3. The next step is to generate the boundary of a half-cylindrical computational domain for the geometry model as shown in Fig. 1. The process accepts both a half and a full (symmetric about the $x-z$ plane $y=0$ ) input surface mesh. It is assumed that the solution is always symmetric; therefore, the surface mesh is always split at this symmetry plane. Once the surface mesh is split, all triangles which have at least one node that lies in the negative direction of the $y$-axis are removed resulting in a half mesh.

The automation process uses a few intuitive input parameters to define the size and the point distribution on each boundary curve of the half-cylindrical computational domain (see Fig. 2). Because the tetrahedral mesh generated by AFLR3 is body-fitted, the point distributions on the surface mesh and on the boundary curves of the half-cylindrical domain can be used to control the resolution of final tetrahedral volume mesh. A current limitation of this approach is that it is not possible to explicitly specify higher mesh refinement at a user-specified region of the computational domain. This can be a problem when performing CFD analysis with powered engine boundary conditions since the refinement of the plume region may not be sufficient to properly capture the flow physics.

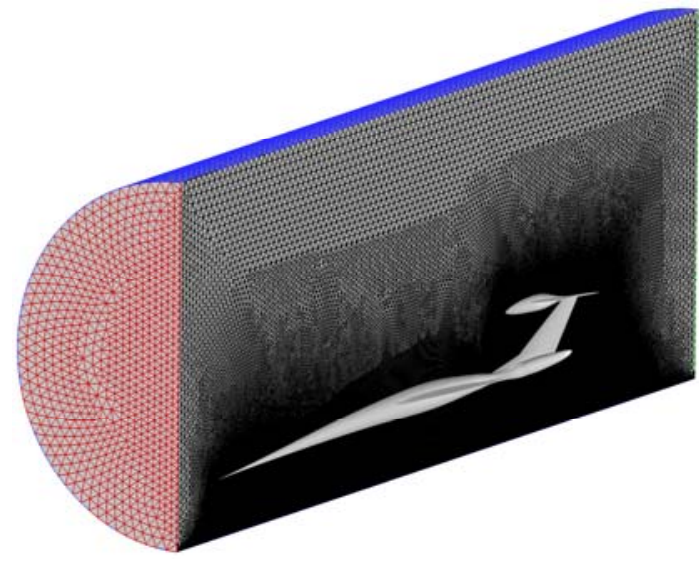

Figure 1. Core mesh boundary of the computational domain.

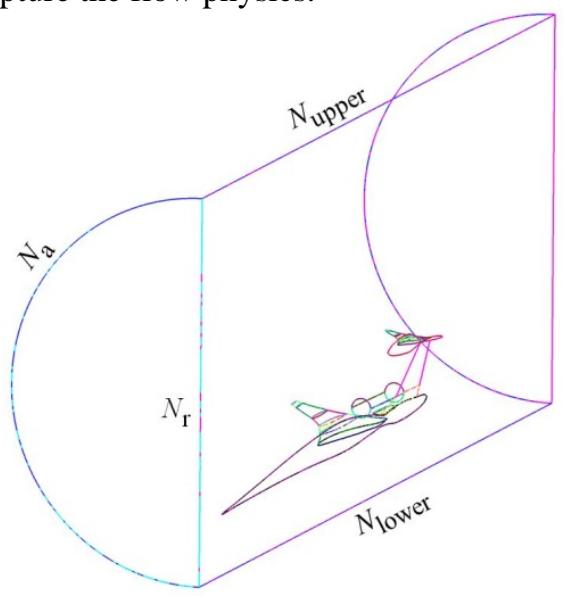

Figure 2. Core boundary mesh point distributions.

The process uses a few additional inputs to define the location of the configuration within the half-cylindrical computational domain (see Fig. 3). These input parameters are ratios of the configuration body length and define the height of the configuration $\left(H_{\mathrm{far}}\right)$ from the under-track (bottom of the half-cylinder), the fore distance of the halfcylinder from the nose of the configuration $\left(X_{\text {fore }}\right)$, and the aft distance of the half-cylinder from the end of the configuration $\left(X_{\text {aft }}\right)$. The half-cylindrical computational domain is automatically sized based on $H_{\text {far }}$ and the radius of the cylinder required to fully enclose the configuration in the spanwise direction. A margin parameter can also be provided to define the distance in the spanwise direction between the configuration wingtip and the half-cylindrical outer boundary.

In addition to the boundary curves of the half-cylindrical computational domain, the set of boundary curves defined by the intersection of the symmetry plane and the surface mesh need to be determined. At this point in the process, the surface mesh is already a half mesh, and therefore, the intersection of the symmetry plane and the surface mesh can be found by determining the sequence of vertices on the triangle edges with no neighboring triangle. Each face of the half-cylindrical domain in Fig. 2 is then meshed using AFLR2 and "stitched" to produce a watertight mesh which defines the boundaries of the computational domain. Note that the meshing process for the fore, aft, and half-cylindrical boundary faces is simple since all boundary curves are defined analytically. However, 
AFLR2 was a key enabler for generating the more complex mesh on the symmetry face of the computational domain. With the boundary mesh created, AFLR3 is then used to generate the "core" volume mesh within this halfcylinder. This core volume mesh is sufficient for aerodynamic analysis.

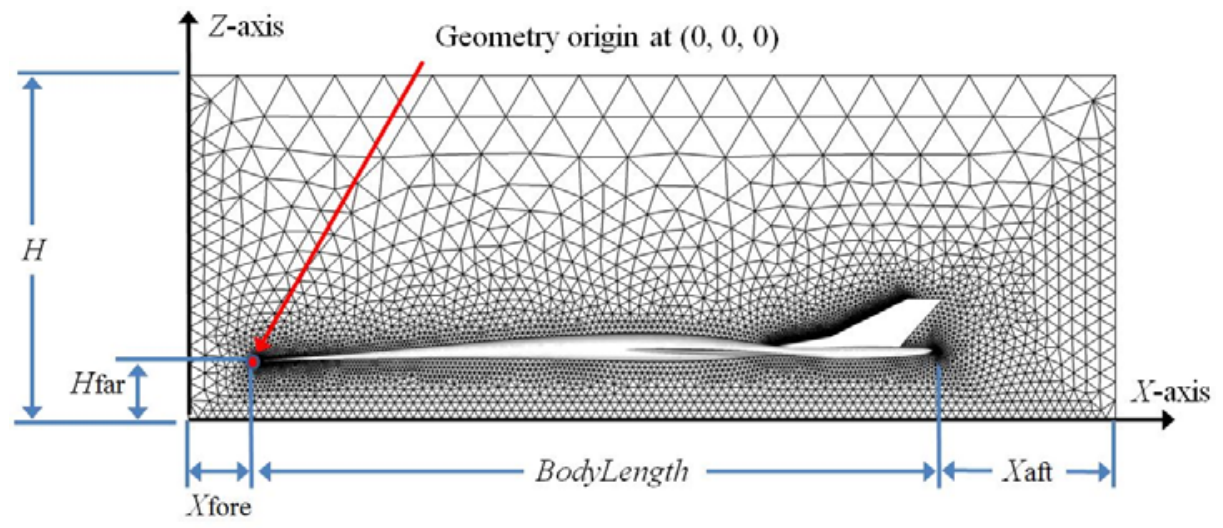

Figure 3. Definition of symmetry mesh for the core boundary.

\section{Generation of Tetrahedral Layer for Off-Body Pressure Calculation}

For CFD-based sonic boom analysis, the off-body pressure has to be calculated several body lengths away from the aircraft. The cost of extending the volume mesh several body lengths for accurate calculation of off-body pressure is not computationally practical. The Boom Grid (BG) $)^{3}$ method is integrated to close this technical gap and allow accurate off-body pressure calculation sufficiently far from the configuration where the 3-D aerodynamic effects are fully resolved.

The BG method is similar to other "extrusion" grid generation approaches in that the new grid is created by extending lines from grid points on a boundary surface of an existing grid in a direction approximately normal to the surface. The new cells are extruded through the outer, typically cylindrical, far-field boundary of an existing core 3D grid around a configuration, creating a "collar" grid (see Fig. 4). For triangular outer boundary faces on the core grid, the extruded cells are prisms that can be used directly in some flow solvers, but often need to be split into tetrahedral cells for other codes. The BG method utilizes very efficient cell generation and splitting algorithms that typically take only a few seconds to generate hundreds of millions of new tetrahedral collar grid cells.

The extension direction is approximately normal to the original surface points when viewed from the front, but the new grid lines are swept in the stream-wise direction. This grid-line shearing, along with stretching of the distance between subsequent layers, creates high-aspect ratio cell faces that are closely aligned with the Mach angle so that the numerical dissipation of the boom signature is reduced. Depending on the desired location for extracting a sonic boom signature, anywhere from 20 to more than 100 layers of cells may need to be generated.

If the outer boundary is a cylinder and the collar grid lines are extruded normal to the original core grid outer boundary, then all of the extruded grid lines appear to originate at the center of the circle (front view of the cylinder) as an azimuthal reference point. If the core grid has the configuration located at this reference point, then boom signature propagation lines will be approximately aligned with the azimuthal grid lines, as desired. The weakness of this approach, however, is that there can be a significant distance from the bottom of the fuselage to the beginning of the collar grid. As the grid is not sheared and stretched in this region, the signal can be dissipated before reaching the collar grid.

If only under-track signatures are needed, then the above problem can be resolved (though with some additional cost due to more grid cells) by simply increasing the radius of the outer core grid cylinder to allow the configuration to be shifted down closer to the lower boundary. For off-track signatures, however, this approach gives poor alignment of the grid lines and signal propagation direction. The BG method attempts to address this problem by using a variable azimuthal reference point instead of the standard center of the circle. This allows the configuration to be shifted down closer to the outer boundary and still have the azimuthal lines that emanate from the configuration for better signal propagation. The azimuthal reference point can be calibrated to focus the grid lines in a desired off-track direction to provide better signal resolution at the off-track angle with very little degradation of the under-track signature. Figure 5 illustrates this capability, showing collar grid inflow planes for standard undertrack and focused off-track cases. Reference 3 provides more detail on this option. 
The BG method is an improvement over the previous SSGRID ${ }^{13}$ and SSG grid stretching and shearing codes in terms of being able to reach a given distance below the aircraft before creating negative volume cells. Further study into the cause of the negative cells has shown that this is an inherent feature for grid extrusion methods if the initial outer grid has surface triangles with a downstream point that does not line up directly behind one of the upstream points. This is illustrated in Fig. 6 for a triangle with all 3 points on the outer cylinder of radius $R$ and with point $\mathrm{C}$ downstream of points A and B by a distance $d x$. Points A and B are off-set from point C by an azimuthal angle $\phi$ above and below $C$, respectively. This off-set moves the two points inward by a distance $d y$ which is given by

$$
d y=R-R \cos (\phi)
$$

resulting in a face inclination angle $\alpha$, computed as

$$
\alpha=\tan ^{-1}(d y / d x)
$$

As layers of cells are added to the collar grid, $d x$ remains fixed, but the value of $R$ increases, thus increasing $d y$ and $\alpha$. Eventually, the boundary face inclination angle becomes parallel to the interior cell faces that are sheared at the Mach angle and the volume of the cell connected to that face becomes negative. A solution to this problem is to generate the core grid outer boundary faces so that one side of each triangle on the half-cylinder is parallel to the $x$ axis. Using this approach, BG has successfully generated collar grids with over 1400 cell layers to distances of 100 body lengths without negative cells.

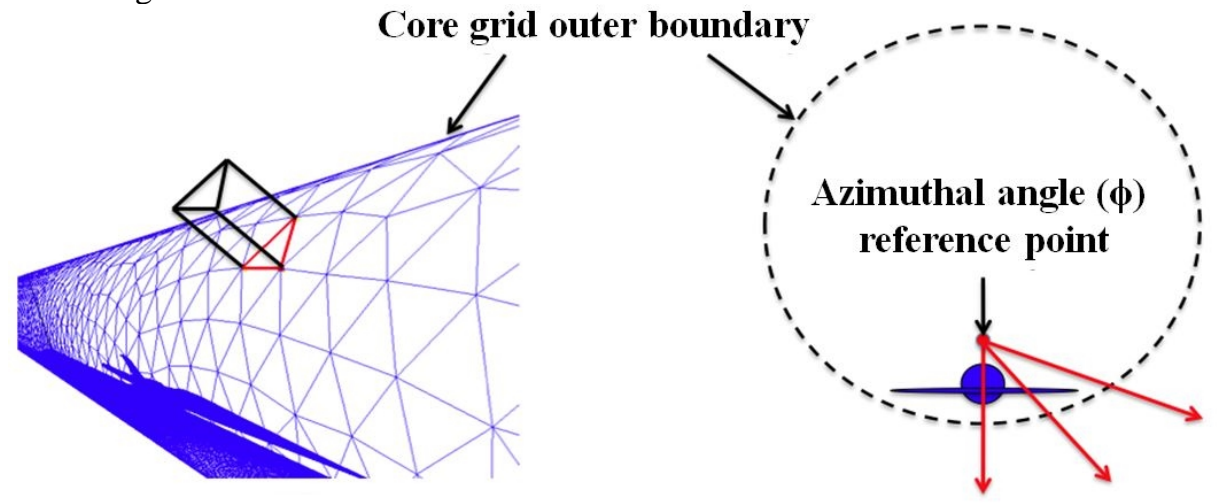

Figure 4. Prism extrusion process in the BG grid methodology.

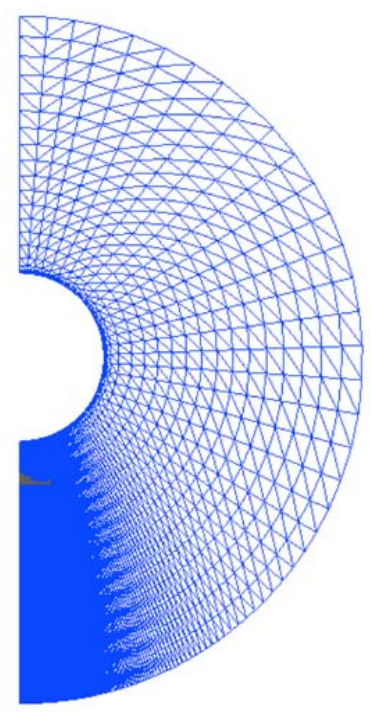

a) Under-track

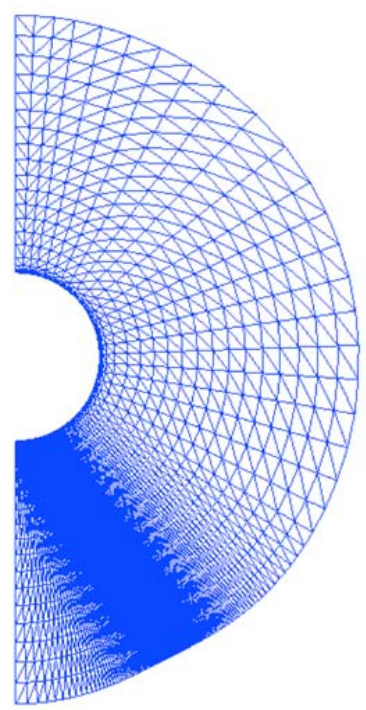

b) Off-track

Figure 5. Effect of BG grid-focusing option for off-track analysis on collar grid inflow plane. 

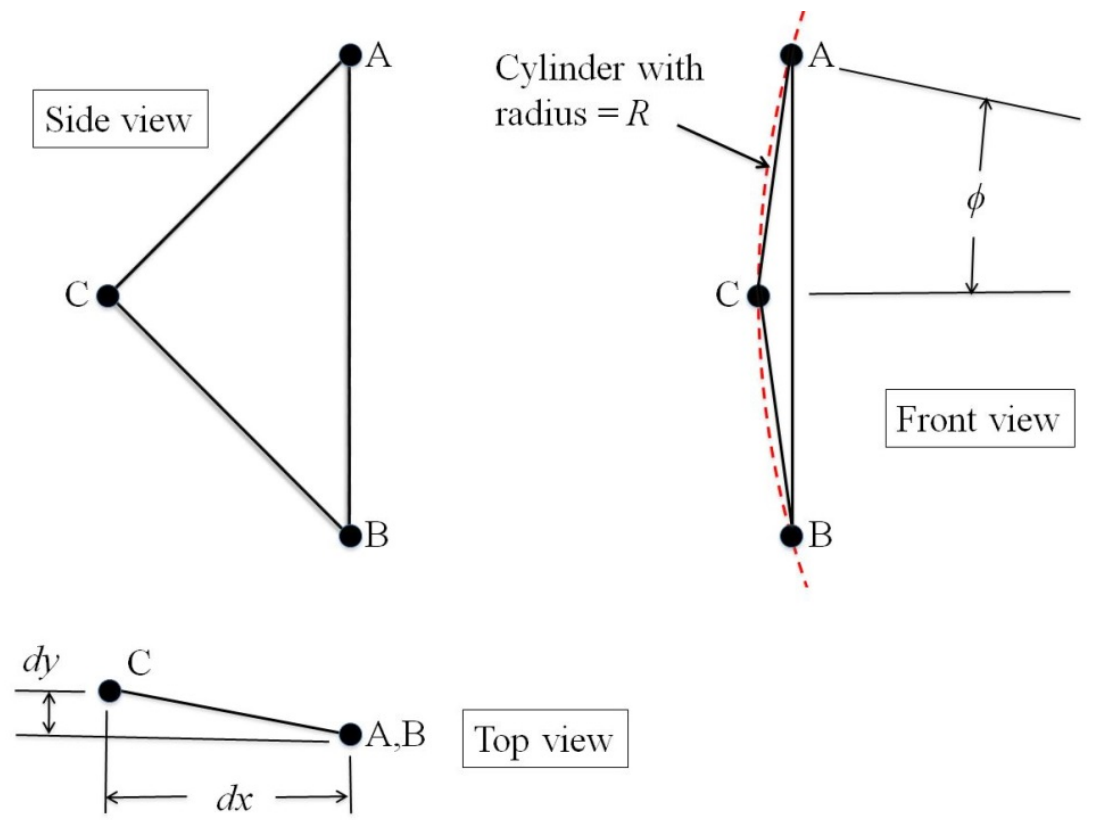

Figure 6. Three-view illustration of non-aligned outer boundary face inclination problem.

\section{Verification Case with a Low-Boom Demonstrator Configuration}

The automated process is used for CFD-based sonic boom analysis of the low-boom demonstrator configuration documented in Ref. 14 and shown in Fig. 7. The low-boom demonstrator is $127 \mathrm{ft}$ in length and contains flowthrough nacelles. Comparison of off-body pressure distribution at 3 body lengths from Cart3D, FUN3D, and USM3D is conducted to understand the code dependency of sonic boom characteristics. The verification of the process is conducted for an inviscid analysis but the method is currently capable of generating grids for inviscid and viscous CFD analyses.

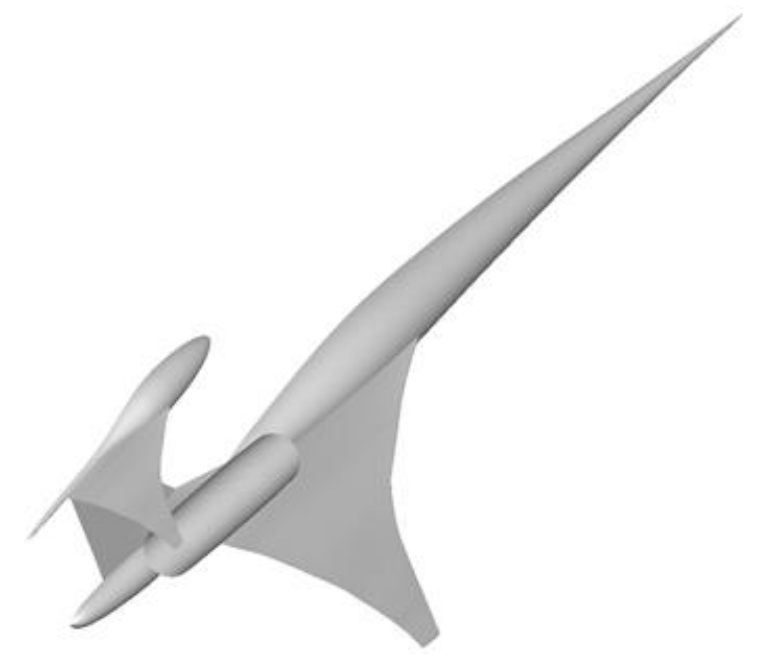

Figure 7. Low-boom demonstrator configuration.

The boundary and volume meshes of the computational domain for the low-boom demonstrator were generated by AFLR2 and AFLR3, respectively. The BG method was then used to extrude the mesh to 3 body lengths (see Fig. 8). On the right hand side of Fig. 8, the symmetry boundary of the core mesh is shown with a black mesh and the extruded collar mesh generated by the BG method is shown by the blue mesh. Note that the flow-through nacelle is not visible in Fig. 8 because it is obscured by the symmetry boundary mesh. This is a relatively coarse mesh consisting of 50 points along the azimuthal direction $\left(N_{\mathrm{a}}\right), 50$ points along the radial direction $\left(N_{\mathrm{r}}\right), 500$ points along the under-track $\left(N_{\text {lower }}\right)$, and 100 points along the over-track $\left(N_{\text {upper }}\right)$. The configuration was located $10 \mathrm{ft}$ above the

American Institute of Aeronautics and Astronautics 
lower boundary of the core mesh and the collar mesh was extruded to 3 body lengths with BG. The flight condition for CFD analysis is set to a Mach number of 1.6 and an angle of attack of 0.6 deg. Note that the shape of the collar mesh is also dependent on the Mach number and angle of attack to ensure that the mesh can capture all shocks up to a distance of 3 body lengths.
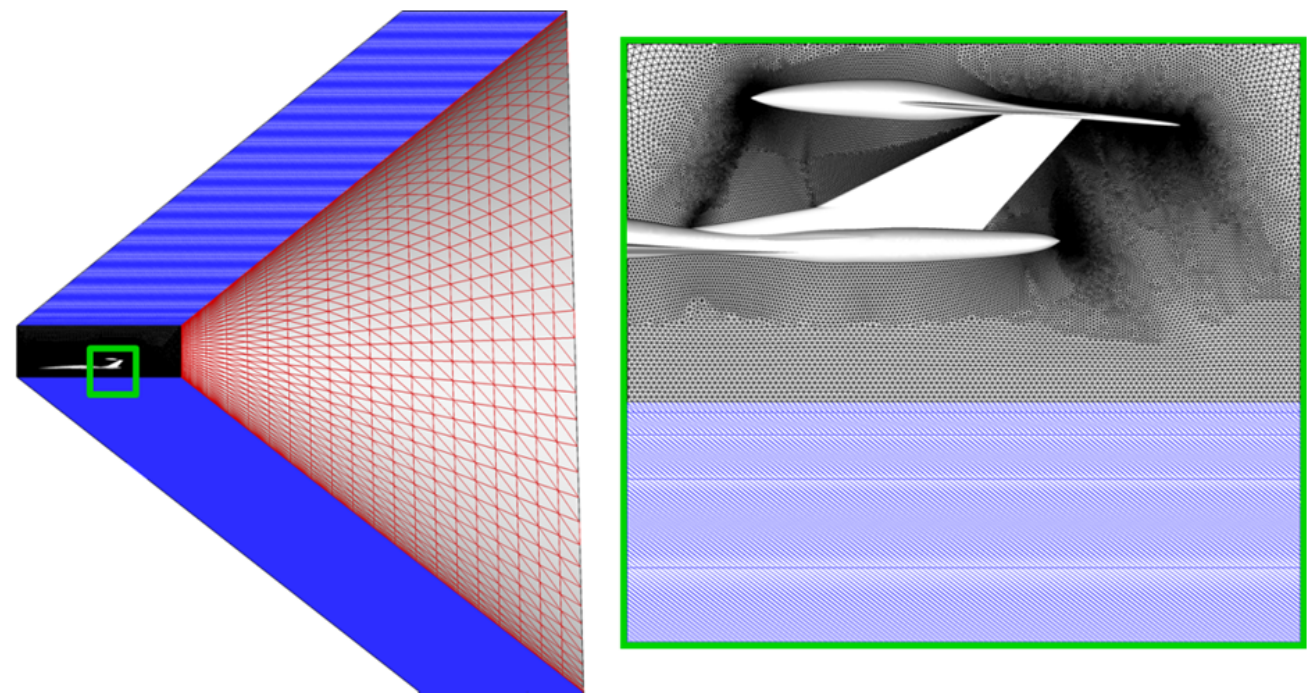

Figure 8. AFLR3 volume mesh and collar for calculation of under-track $d p / p$ at 3 body lengths.

The verification study is conducted through comparison with different CFD analysis codes (Cart3D, FUN3D, and USM3D), and different meshing strategies. The van Leer limiter is used in the CFD analysis with Cart3D and FUN3D, and the min-mod limiter is used with USM3D. Two additional CFD meshing strategies are used to verify the AFLR3 mesh. The alternative meshing strategies include a VGRID-generated mesh that is stretched and sheared with the SSGRID method and a Cart3D adjoint-based adapted Cartesian mesh.

The off-body pressure distribution comparison at 3 body lengths is shown in Fig. 9. The first two curves (blue and red) provide a comparison of the off-body pressure computed with FUN3D for the VGRID- and AFLR3generated meshes. These show good agreement in shock structure with the main difference being the greater shock resolution of the AFLR3 mesh. Note that the FUN3D pressure distribution with the AFLR3-generated mesh shows some characteristics associated with the "sonic glitch"15 along the fore ramp. This manifests as small oscillations and are a result of very close alignment between the off-body grid and the Mach wave. This can be resolved by providing a small offset angle to the grid and Mach wave alignment. The second and fourth curve (red and black) provide a comparison of different CFD analyses (FUN3D and USM3D) for the same AFLR3-generated mesh. Once again, the two curves show similar shock structure with the USM3D solution appearing more dissipated. This higher dissipation is likely due to the use of the more dissipative Min-mod limiter in USM3D. Lastly, the pressure distribution calculated with Cart3D with an adjoint-based adapted mesh is given by the third curve in green. Here, the cost function for the Cart3D adjoint-based mesh adaptation places more emphasis in the resolution of the larger shocks, while smaller shocks appear more dissipated. More importantly, there appears to be a significant discrepancy in the shock location at approximately $590 \mathrm{ft}$. This shock location discrepancy between FUN3D/USM3D and Cart3D also appears when the Cart3D analysis is conducted with a non-adapted, Mach-aligned mesh, as well as other configurations which are not presented here. The cause of this discrepancy cannot yet be explained and further investigation is needed. However, it is not believed to be associated with the automated tetrahedral meshing process that has been presented because it appears to be dependent on the CFD solver and not on the meshing strategy. 


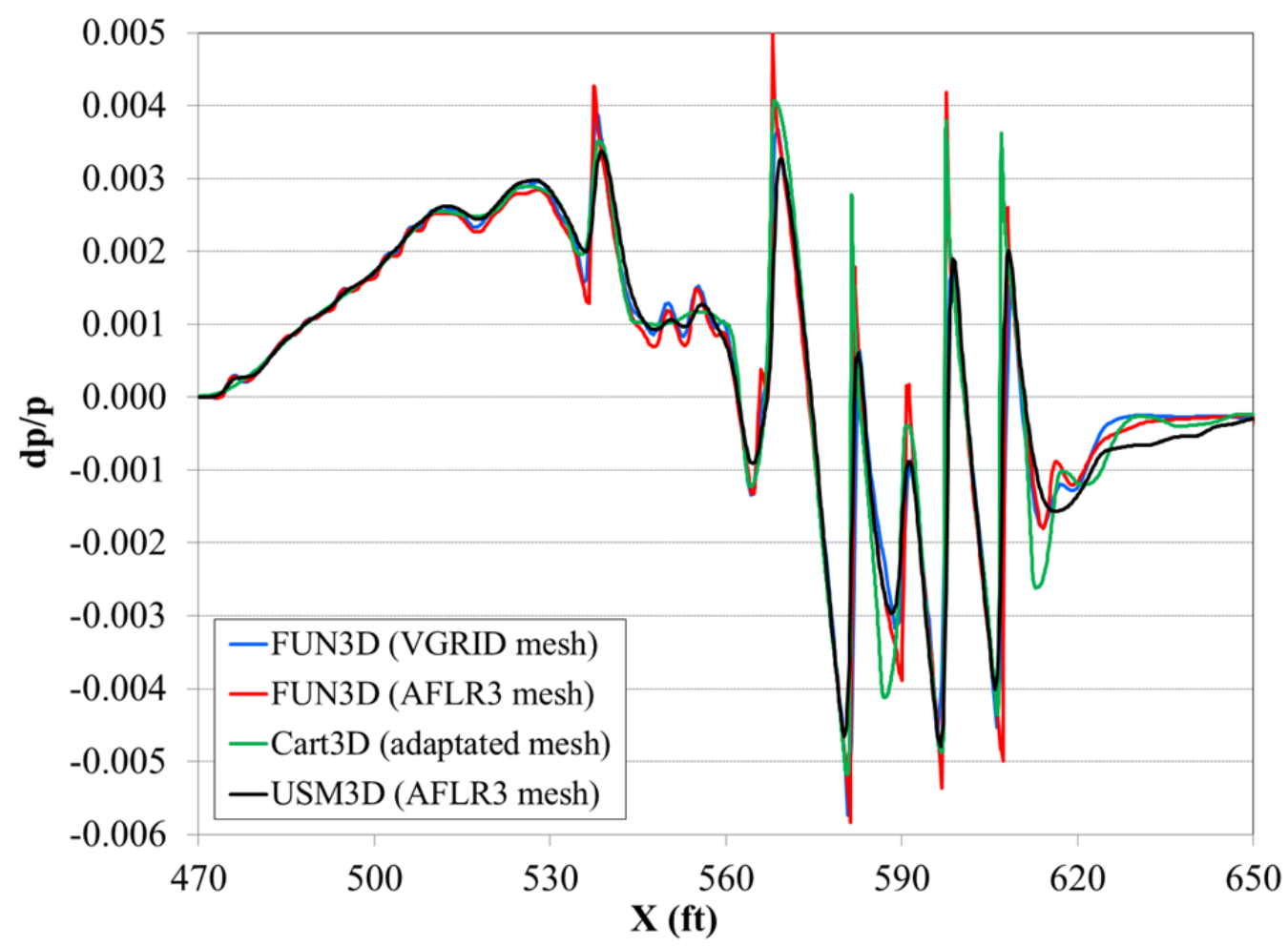

Figure 9. Comparison of under-track pressure distribution at 3 body lengths.

\section{Summary}

An automation process has been presented for the generation of tetrahedral meshes for CFD analysis starting with a surface triangulation of reasonable quality. Although the process was developed for CFD-based sonic boom analysis and design of supersonic configurations, it can also be applied to aerodynamic analysis of aircraft configurations in any flight regime.

The automation process leverages existing surface-surface intersection methods to define the surface mesh. This allows CFD analysis of more complex configurations because it eliminates the limitation of an earlier process documented in Ref. 11, which required the intersection of two components to form a closed loop on each component. The process also leverages the advancing front and local reconnection methods in AFLR2 and AFLR3 to generate the boundary and volume mesh of the core computational domain. The BG method is integrated to perform the extrusion of the mesh collar for accurate calculation of the off-body pressure distribution several body lengths away from the aircraft.

Lastly, a verification case using a low-boom configuration has been presented for comparison of the under-track off-body pressure distribution at 3 body lengths. The verification highlights the differences between three meshing strategies and three well-established CFD analysis codes. The process generated an off-body pressure distribution consistent with other meshing strategies. A discrepancy was found in one shock location that appears to be dependent on the CFD code instead of the meshing strategy. Nevertheless, this shock location discrepancy is still under investigation.

\section{Acknowledgment}

The authors would like to thank Mathias Wintzer (Analytical Mechanics Associates) for providing a Cart3D verification solution for the low-boom demonstrator and Sriram K. Rallabhandi (National Institute of Aerospace) for providing feedback that contributed to improvement in the robustness and operability of the automation process.

\section{References}

${ }^{1}$ Open VSP, http://www.openvsp.org, [cited May 2013].

${ }^{2}$ AFLR3, http://www.simcenter.msstate.edu/docs/aflr3/, [cited May 2013].

${ }^{3}$ Nayani, S. N., and Campbell, R. L., "Evaluation of Grid Modification Methods for On- and Off-Track Sonic Boom Analysis”, AIAA Paper 2013-0798, January 2013. 
${ }^{4}$ FUN3D, http://fun3d.larc.nasa.gov/index.html, [cited May 2013].

${ }^{5}$ USM3D, http://tetruss.larc.nasa.gov/usm3d/, [cited May 2013].

${ }^{6}$ Geiselhart, K. A., Ozoroski, L. P., Fenbert, J. W., Shields, E. W., and Li, W., "Integration of Multifidelity Multidisciplinary Computer Codes for Design and Analysis of Supersonic Aircraft,” AIAA Paper 2011-465, January 2011.

${ }^{7}$ Ordaz, I. and Li, W., "Integration of Off-Track Sonic Boom Analysis in Conceptual Design of Supersonic Aircraft,” AIAA Paper 2011-464, January 2011.

${ }^{8}$ Cart3D, http://people.nas.nasa.gov/ aftosmis/cart3d/cart3Dhome.html, [cited May 2013].

${ }^{9}$ Gridgen, http://www.pointwise.com/gridgen/, [cited May 2013].

${ }^{10}$ VGRID, http://tetruss.larc.nasa.gov/vgrid/, [cited May 2013].

${ }^{11}$ Li, W., Campbell, R. L., Geiselhart, K. A., Shields, E. W., Nayani, S., and Shenoy, R., "Integration of Engine, Plume, and CFD Analyses in Conceptual Design of Low-Boom Supersonic Aircraft,” AIAA Paper 2009-1171, January 2009.

${ }^{12}$ McDonald, R., "VSP File Types and VSP Meshing,"

http://www.openvsp.org/wiki/lib/exe/fetch.php?media=file_type_meshing_2013.pdf, [cited November 2013].

${ }^{13}$ Campbell, R. L., Carter, Melissa B., Deere, Karen A., and Waithe, K. A., "Efficient Unstructured Grid Adaptation Methods for Sonic Boom Prediction”, AIAA Paper 2008-7327, August 2008.

${ }^{14}$ Ordaz, I. and Li, W., “Adaptive Aft Signature Shaping of a Low-Boom Supersonic Aircraft Using Off-Body Pressures,” AIAA Paper 2012-0020, January 2012.

${ }^{15}$ Moschetta, J.-M., and Gressier, J., “The sonic point glitch problem: A numerical solution”, Lecture Notes in Physics, Vol. 515, pp. 403-408., 1998. 